\title{
A Case of Cutaneous Tuberculosis Presenting with Generalized Xanthogranulomas
}

\author{
Leah Antoinette M. Caro-Chang ${ }^{1}$ and Eileen Liesl A. Cubillan² \\ ${ }^{1}$ Section of Dermatology, Department of Medicine, Philippine General Hospital, University of the Philippines Manila \\ ${ }^{2}$ Section of Dermatology, Department of Medicine, College of Medicine and Philippine General Hospital, University of the Philippines Manila
}

\begin{abstract}
A 33-year-old male had a 22-year history of generalized xanthogranulomas but had a normal lipid profile. He also developed ulcerating plaques on his arm and back that were consistent with cutaneous tuberculosis on histopathologic and polymerase chain reaction PCR studies. In a normolipemic patient with generalized xanthogranulomas, a reactive granulomatous response of histiocytes to infection is explored since these share a common CD 14+ precursor with the macrophages that are vital in tuberculosis.
\end{abstract}

Key Words: xanthogranuloma, tuberculosis, histiocytes

\section{INTRODUCTION}

Multiple xanthogranulomas in adults are unusual, more so in the setting of a normal lipid profile. Considered a variant of non-Langerhans cell histiocytosis, adultonset xanthogranulomas have been reported to occur in association with various diseases but the exact etiology remains under investigation. It is proposed to be a reactive granulomatous response to infection, physical stimuli and other unknown stimuli. ${ }^{1}$ Most case reports of adult xanthogranulomas with multiple disseminated lesions were associated with hematologic diseases such as leukemia and lymphoma. ${ }^{2}$ Other associations reported in the literature are with gastrointestinal stromal tumor, ${ }^{3}$ diabetes mellitus, ${ }^{4,5}$ and an increase in the number of lesions after trauma and surgery. ${ }^{4}$ This is the first case reporting generalized xanthogranulomas associated with tuberculosis.

\section{CASE}

This is a case of a 33-year old male with a 22-year history of multiple skin colored papules on his legs that were very pruritic. The papules were waxing and waning for several years. The eruption of lesions was accompanied by undocumented fever. Eight years prior to consult, the patient developed a very pruritic, firm, skin-colored plaque on the right antecubital fossa which subsequently developed purulent discharge and occasional bleeding. The lesion spontaneously resolved after four months with the development of similar lesions on his back and left forearm. At that time, there was a recurrence of the pruritic papules that developed a yellow hue noted on the trunk and eventually spreading to all extremities, face, and scalp. The patient was diagnosed with pulmonary tuberculosis seven

Section of Dermatology

Department of Medicine

Philippine General Hospital

University of the Philippines Manila

Taft Avenue, Manila 1000, Philippines

Telephone: +6325348400 local 5105/5106

Email: Imcaro@up.edu.ph 
years prior to consultation and completed nine months of treatment but the skin lesions persisted.

Physical examination showed generalized erythematous to yellow papules, some with hemorrhagic crusting and yellow plaques (Figure 1). There were cervical, axillary, and
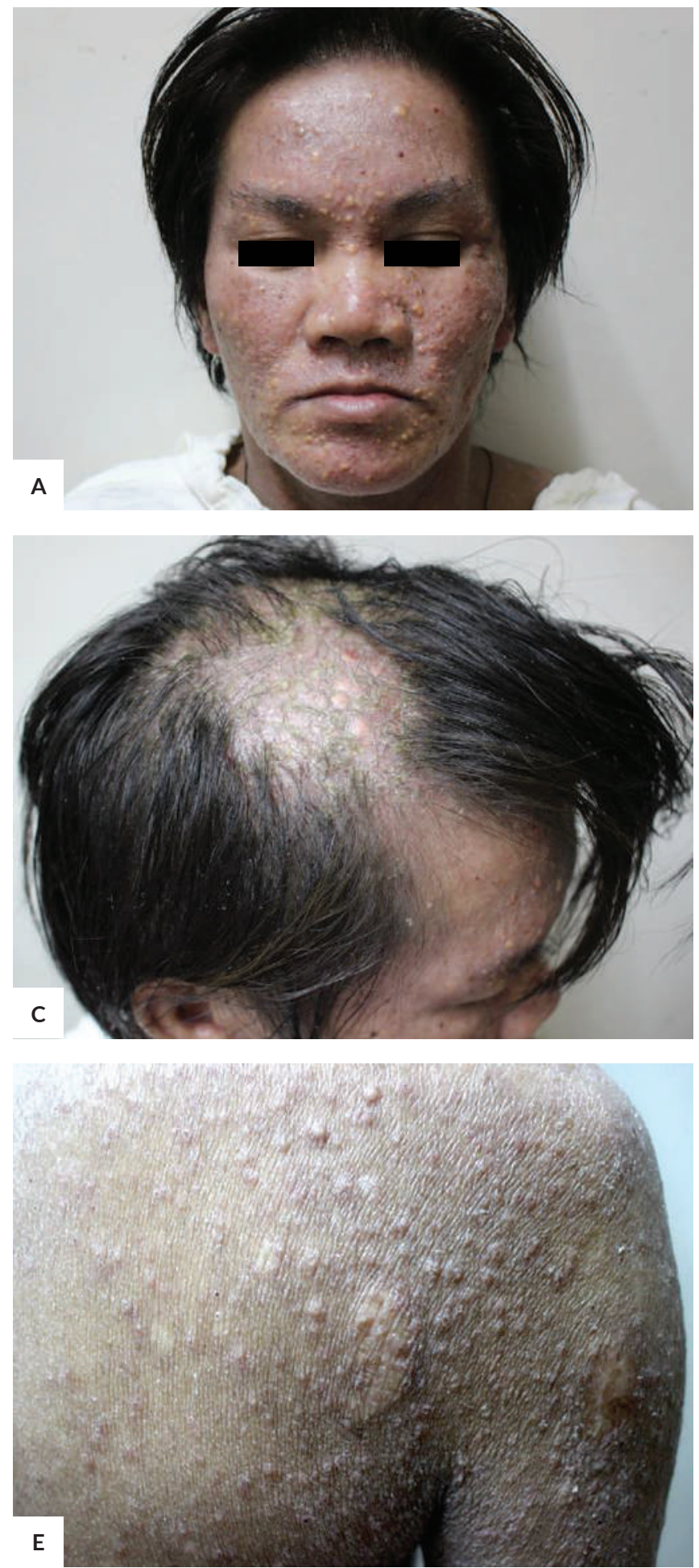

inguinal lymphadenopathies that were firm, non-movable, and non-tender. Dermoscopy of the papules showed a yellow lesion with white globules and surrounding erythema with some telangiectasias (Figure 2). The yellow papule on the face and the skin-colored papule on the arm showed
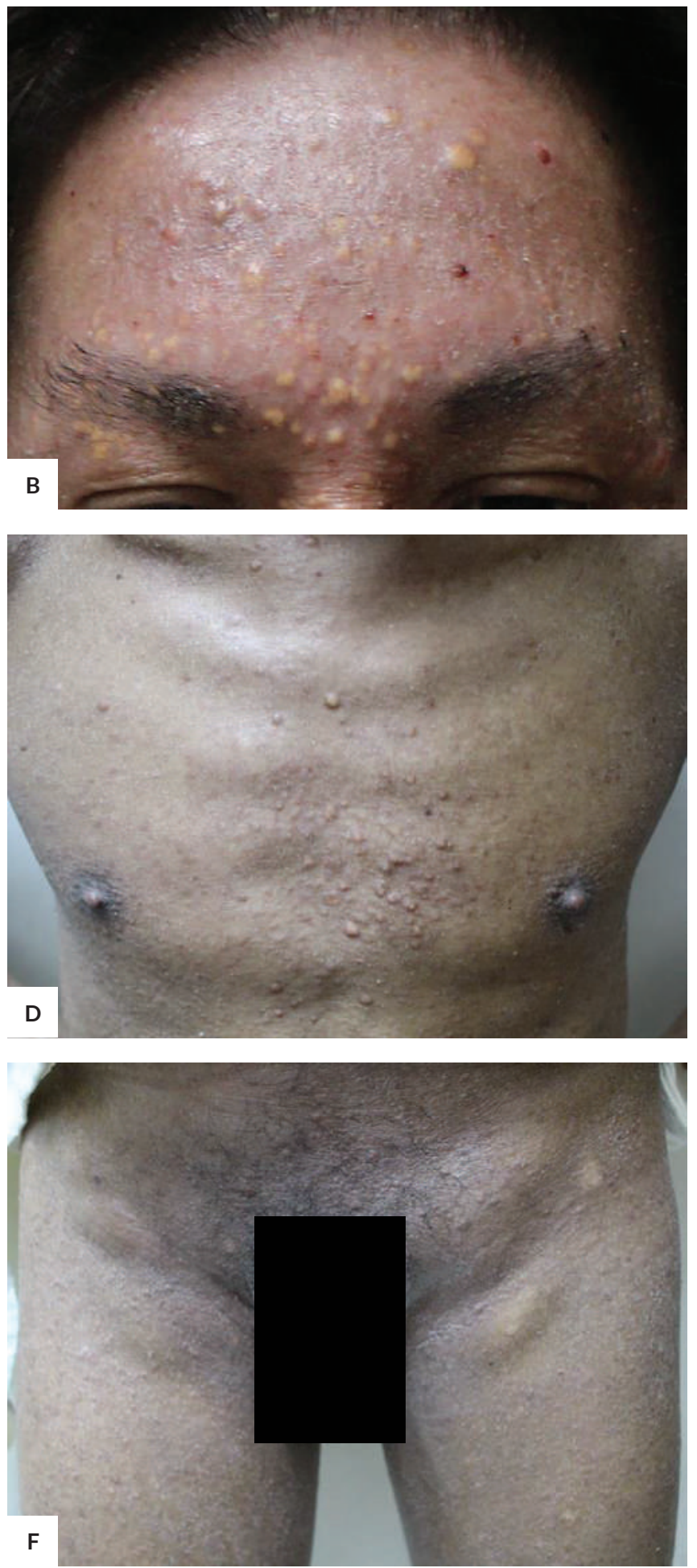

Figure 1. Multiple skin-colored, erythematous and yellow papules on the A and B - face, C - scalp, D - chest, E - back and arm, $\mathrm{F}$ - pubic region with multiple inguinal lymphadenopathy. 
diffuse infiltrates of foamy histiocytes, lymphocytes, and neutrophils with Touton giant cells, giving a histologic diagnosis of xanthogranulomas (Figure 3 ).

Skin-colored to erythematous plaques with areas of atrophy and ulcerations with hemorrhagic and seropurulent crusting were noted on the upper back and left arm (Figure 4). Histologically, the plaque on the left arm showed diffuse infiltrates of foamy histiocytes, lymphocytes, neutrophils and abundant eosinophils (Figure 5). This was sent for Fite staining where a few acid-fast bacilli were noted. The other two specimens, however, were negative for acid fast baciili on Fite stain. Tuberculosis Polymerase
Chain Reaction (TB PCR) (no quantification), was positive. Slit-skin smear was negative on all six sites, making a diagnosis of leprosy unlikely. Tissue studies of the plaque on the left arm showed no growth of fungi on fungal culture, no growth on mycobacterial culture after six weeks of incubation and positive for Acinetobacter baumannii that was considered to be a contaminant and not a real pathogen.

Lipid profile (total cholesterol, triglycerides, HDL, LDL, and VLDL) was normal. Fasting blood sugar, renal and liver function tests and electrolytes were all within normal limits. There was a note of slight anemia with a hemoglobin of $137 \mathrm{mg} / \mathrm{dL}$ at baseline and retained the
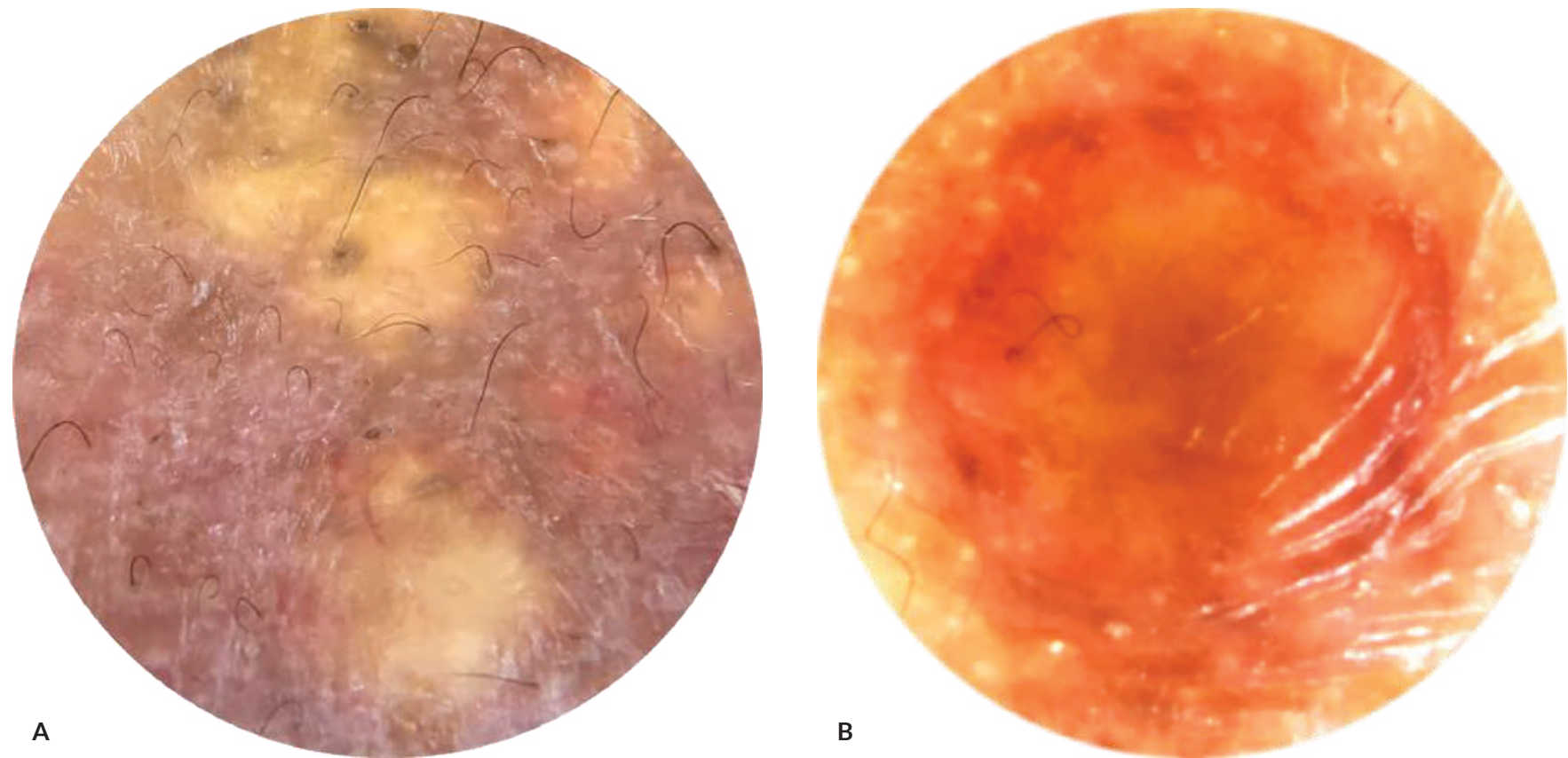

Figure 2. A and B - Dermoscopy of the xanthogranulomas showing a yellow lesion with white globules and surrounding erythema with some telangiectasias.
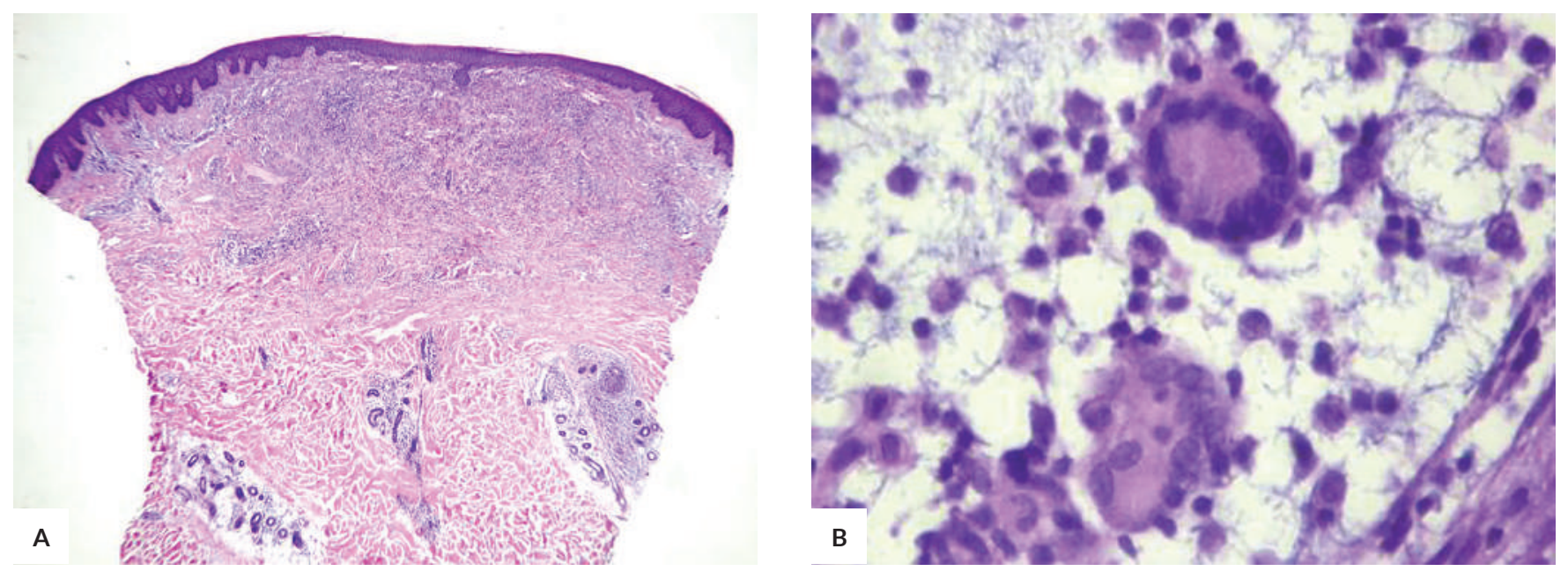

Figure 3. Biopsy of a papule on the arm showing $\mathbf{A}$ - diffuse infiltrates in the superficial dermis composed of $\mathbf{B}$ - Touton giant cells with lymphocytes, histiocytes and eosinophils as seen on higher magnification. Findings consistent with xanthogranulomas. 

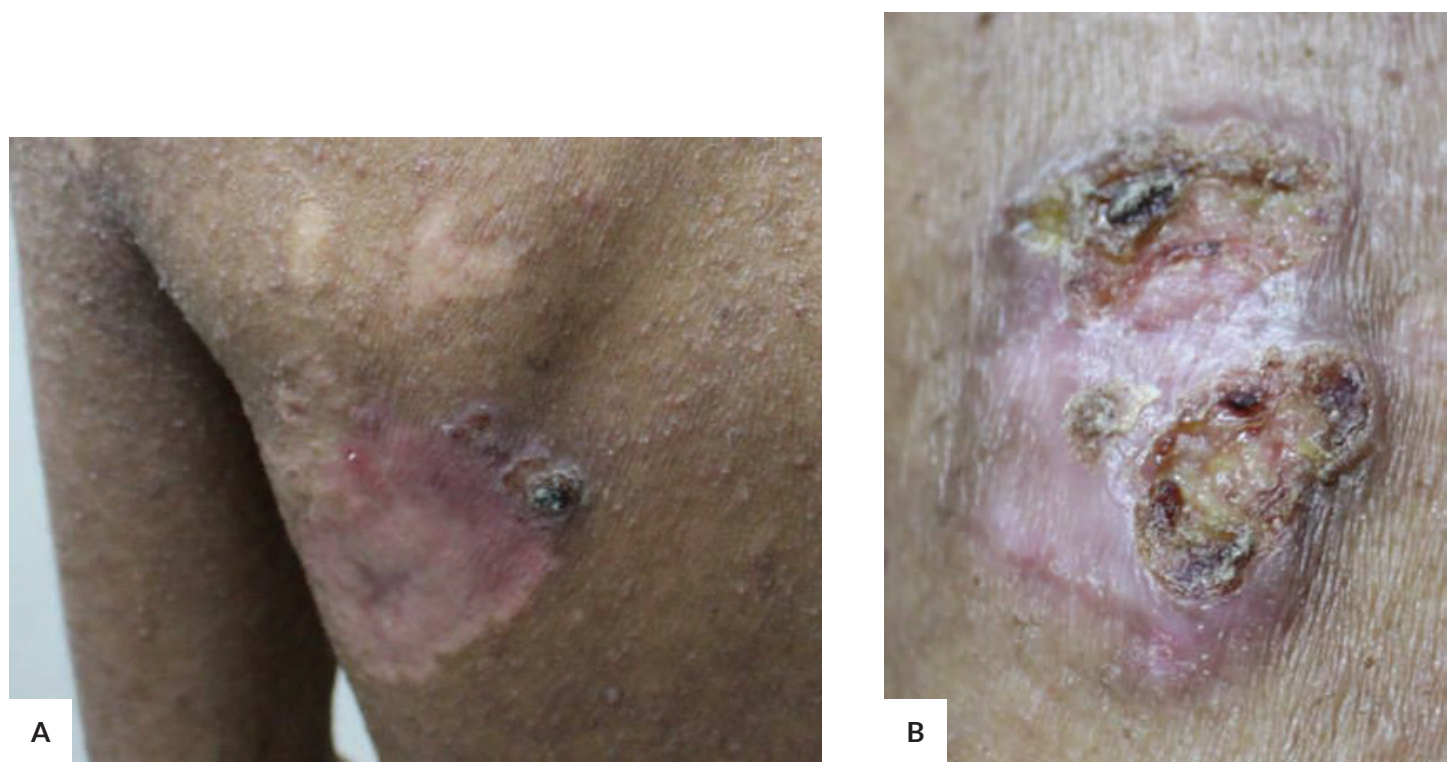

Figure 4. Ulcerated plaques with areas of atrophy, hemorrhagic crusting, seropurulent discharge on A- back and B - left arm.
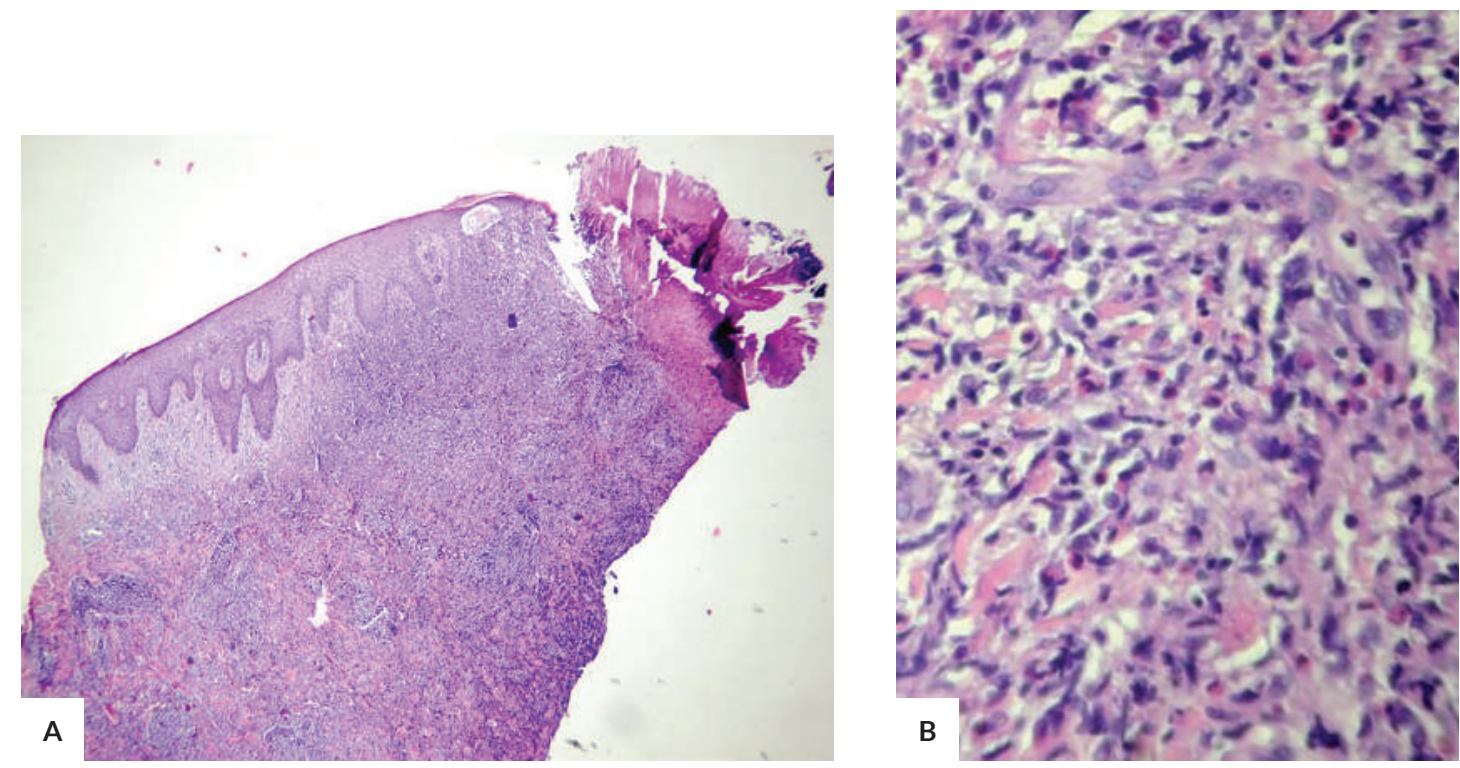

Figure 5. Biopsy of the plaque on the forearm showing $A$ - ulceration and diffuse infiltrates composed of B - foamy histiocytes, lymphocytes and eosinophils. Findings consistent with granulomatous dermatitis.

same value after three months. This was attributed to chronic disease. The Hematology service was not highly considering a blood dyscrasia or a hematologic malignancy. Eosinophilia at 0.11 on initial testing was also noted. Fecalysis was done but did not show any ova or parasites. The value went down after three months at 0.06 . Monocytes were also elevated initially at 0.12 but showed a decline at 0.10 after three months. Urinalysis was also unremarkable. HIV ELISA was nonreactive.

The chest radiograph showed reticulonodular opacities in both upper lobes with associated super traction of the left hilar structures. These findings were suggestive of pulmonary tuberculosis with cicatricial changes. Sputum Gene Xpert received a specimen with salivary appearance where Mycobacterium Tuberculosis (MTB) was not detected.

In order to ensure that there was no underlying malignancy, the hematology specialist advised the patient to undergo a holoabdominal ultrasound and biopsy of the inguinal lymph node care of General Surgery section. The patient had normal ultrasound results while core-needle biopsy of the lymph nodes showed infiltrates of eosinophils and plasma cells consistent with chronic inflammation. 


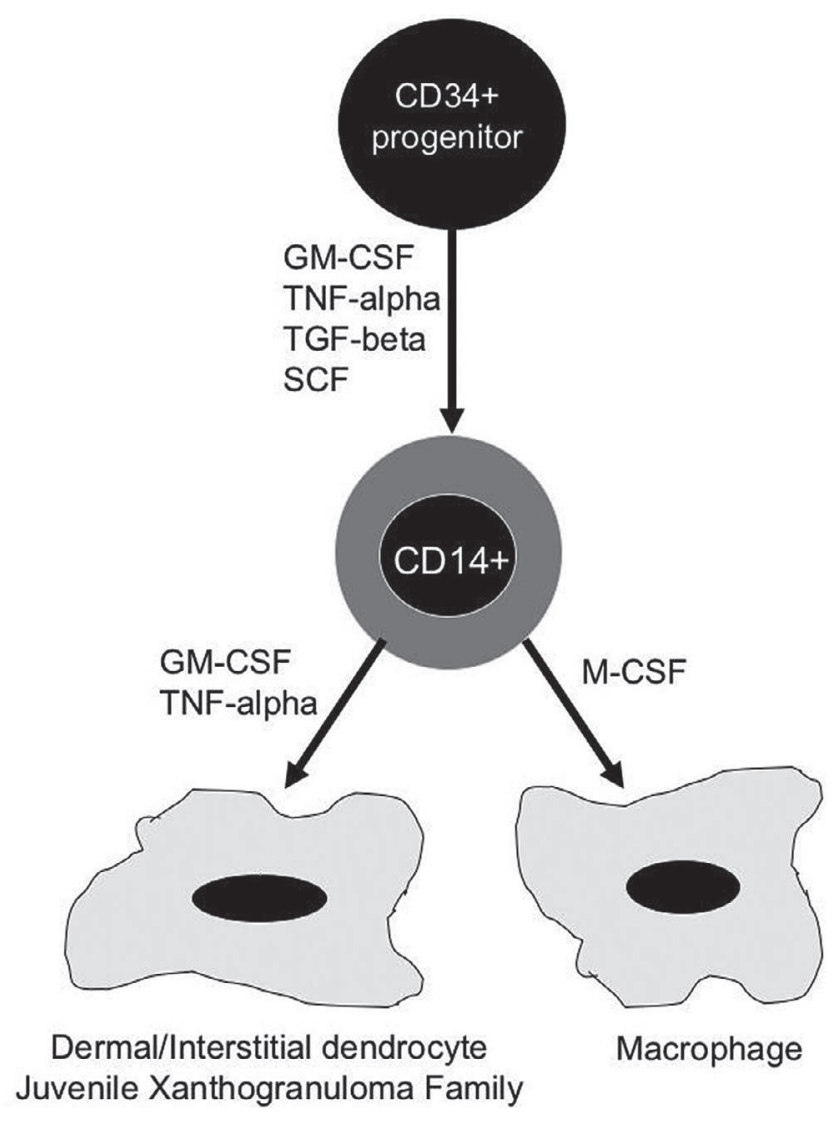

Figure 6. Common histiocytic precursor of the dendrites and macrophages. ${ }^{6}$

With the aid of the ancillary tests and comanagement of other services, the patient was diagnosed with xanthogranulomas and cutaneous tuberculosis (lupus vulgaris). He was started on isoniazid-rifampicinpyrazinamide-ethambutol daily for six months and streptomycin 1gram IM daily for 56 doses. The treatment with anti-tuberculous medications showed a decrease in the size of the ulcer on the plaques on the back and arm. As with other cases of adult xanthogranulomas, the patient's lesions were persistent. However, no new lesions developed.

\section{DISCUSSION}

The connection between xanthogranulomas and tuberculosis may be attributed to the role of histiocytes in both disease entities. The histiocytes or dendrocytes involved in the formation of xanthogranulomas share a common $\mathrm{CD} 14+$ precursor with the macrophages that are integral in the immune response to tuberculosis. Cytokines, such as GM-CSF and TNF-alpha, are needed to stimulate their production. ${ }^{1,6}$ In patients with tuberculosis, levels of IFN-y, TNF alpha, GM-CSF are higher than those in a healthy person $^{7}$ and this, perhaps, provides the stimulus for the continued production of dendrocytes found in xanthogranulomas. This pathway is depicted in Figure 6. It is important to note that the patient was previously managed as a case of pulmonary tuberculosis, so the waxing and waning lesions that appeared prior to diagnosis may have been due to an underlying tuberculosis infection. It is also this history of pulmonary tuberculosis that predisposed him to develop extrapulmonary tuberculosis in the form of cutaneous tuberculosis, consequently perpetuating the reactive granulomatous response in the form of xanthogranulomas.

Juvenile xanthogranulomas spontaneously resolve. Unfortunately, adult xanthogranulomas have a persistent course. ${ }^{6}$ As with our patient, anti-tuberculosis medications did not decrease the number of lesions but, no new lesions have developed since the start of the treatment.

In an adult patient presenting with generalized xanthogranulomas, especially in the setting of a normal lipid profile, it is imperative to perform the proper diagnostic work-up to rule out an underlying illness that may be causing the reactive granulomatous response. Majority of the case reports have identified associations with hematologic malignancies. This is the first case that reported an association with tuberculosis. Therefore, tuberculosis, together with other infectious disorders, should also be investigated in patients presenting with generalized xanthogranulomas.

\section{Statement of Authorship}

All authors approved the final version submitted.

\section{Author Disclosure}

All authors declared no conflicts of interest.

\section{Funding Source}

This paper was funded by the authors. No external funding agency.

\section{REFERENCES}

1. Chiou C, Wang P, Yang L, Kuo T, Hong H. Disseminated xanthogranulomas associated with adult T-cell leukaemia/lymphoma: A case report and review the association of haematologic malignancies. J Eur Acad Dermatology Venereol. 2007;21(4):532-5.

2. Narváez-Moreno B, Pulpillo-Ruiz A, De Zulueta-Dorado T, Conejo-Mir J. Disseminated juvenile xanthogranuloma associated with follicular lymphoma in an adult: successful treatment with chemotherapy and rituximab. a review of the literature. Actas Dermosifiliogr. 2012;104:1-5.

3. Vadeboncoeur S, Provost N. Multiple xanthogranulomas in an adult: known entity, new association. J Cutan Med Surg. 2016;20(5):474-7.

4. Ayvaz H, Celik G, Gönül M, Kiliç A, Özcan N, Çolak A. A case of adult onset disseminated juvenile xanthogranuloma. Our Dermatol Online. 2016;7(1):66-8.

5. Saad N, Skowron F, Dalle S, Forestier J, Balme B, Thomas L. Multiple adult xanthogranuloma: case report and literature review. Dermatology. 2005;212(1):73-6.

6. Weitzman S, Jaffe R. Uncommon histiocytic disorders: The non-Langerhans cell histiocytoses. Pediatr Blood Cancer. 2005;45(3):256-64.

7. Dias M, Bernardes F, Quaresma M, do Nascimento L, Nery JA, Azulay D. Update on cutaneous tuberculosis. An Bras Dermatol. 2014;89(6):925-38. 